Published in final edited form as:

Gastroenterol Clin North Am. 2016 March ; 45(1): 1-8. doi:10.1016/j.gtc.2015.10.004.

\title{
Advances in Management of Acute Pancreatitis
}

\author{
Nigeen $\mathbf{H}$. Janisch, MD and \\ Geisel School of Medicine at Dartmouth, Hanover, NH USA \\ Timothy B. Gardner, MD, MS [Associate Professor] \\ Geisel School of Medicine at Dartmouth, Hanover, NH USA \\ Nigeen H. Janisch: nigeen.h.janisch.med@dartmouth.edu
}

\begin{abstract}
This chapter will review advances in the management of acute pancreatitis. Medical treatment has been primarily supportive for this diagnosis in the past, and despite extensive research efforts, there are no pharmacologic therapies that improve prognosis. The current mainstay of management, notwithstanding the ongoing debate regarding the volume, fluid type, and rate of administration, is aggressive intravenous fluid resuscitation. While in the past antibiotics were used consistently for prophylaxis in severe acute pancreatitis to prevent infection, they are no longer used unless infection is documented. Enteral nutrition, especially in patients with severe acute pancreatitis, is considered a cornerstone in management of this disease.
\end{abstract}

\section{Keywords}

Acute pancreatitis; medical management; fluid resuscitation; antibiotics; walled-off pancreatic necrosis; BiSAP; prognostication

\section{Introduction}

Acute pancreatitis is a frequently devastating pancreatic inflammation that has been associated with significant morbidity, mortality, and hospitalization costs [1]. The incidence of acute pancreatitis has been increasing and features an overall mortality rate of $5 \%$ that may be as high as $30 \%$ in the most severe cases [2-4]. It was the most common inpatient gastrointestinal diagnosis in 2009, totaling over 270,000 United States hospitalizations and incurring costs of over 2.6 billion dollars [5]. The updated Atlanta classification divides acute pancreatitis into mild and severe types [6,7]. Mild, characterized by pancreatic inflammation without necrosis or organ failure, is known as interstitial edematous pancreatitis, which is usually self-limiting and resolves in about one week. Severe

Corresponding Author: Timothy B. Gardner MD MS, Associate Professor of Medicine, Section of Gastroenterology and Hepatology, Dartmouth Hitchcock Medical Center, One Medical Center Drive, Lebanon, NH 03756, 603-650-6472 (p) 603-650-5225 (f), timothy.b.gardner@hitchcock.org.

Disclosures: The authors have no disclosures

Publisher's Disclaimer: This is a PDF file of an unedited manuscript that has been accepted for publication. As a service to our customers we are providing this early version of the manuscript. The manuscript will undergo copyediting, typesetting, and review of the resulting proof before it is published in its final citable form. Please note that during the production process errors may be discovered which could affect the content, and all legal disclaimers that apply to the journal pertain. 
pancreatitis, occurring in about $20 \%$ of cases, predisposes to local complications such as pancreatic necrosis, abscess formation, and pseudocysts. Severe pancreatitis is subdivided further into moderate and severe depending on the presence and duration (> 48 hours) of organ failure. In this chapter, we will detail treatment of acute pancreatitis, including highlighting new insights into prognostication and focusing on intravenous fluid resuscitation and the current evidence behind the use of antibiotics and pharmacologic therapies.

\section{Predicting Severity}

- Simple, universally obtainable markers such as the change in BUN level are equally predictive of severity when compared with more complicated systems

Predicting the severity of acute pancreatitis can be challenging. Since 1974, when the Ranson's criteria were first proposed, multiple scoring systems (APACHE-II, BiSAP, Marshall Score, etc.) have been developed as a means of improving the ability to predict severity in acute pancreatitis. However, despite the use of these often complex systems, laboratory abnormalities in hematocrit, creatinine, and blood urea nitrogen (BUN) can be used as effective prognostic indicators with equivalent accuracy. For example, an increased risk of pancreatic necrosis has been linked with an elevated hematocrit at admission or within the first 24 hours, as well as an elevated creatinine level within the first 48 hours [8-10]. With regards to BUN, A 2011 meta-analysis of 1043 acute pancreatitis cases demonstrated that a BUN $\geq 20 \mathrm{mg} / \mathrm{dl}$ (OR 4.6, 4.3 respectively) at admission, or an increase in levels within the first 24 hours, was associated with an increased risk of mortality and death [11]. Thus, it is recommended that a simple marker, such as BUN, be used as means of assessing severity and potential progression to organ failure.

\section{Fluid Resuscitation}

- Goal to decrease hematocrit and BUN within the first 24 hours of hospitalization.

- Early aggressive fluid resuscitation with $250-500 \mathrm{~mL}$ per hour of isotonic crystalloid in the first 12-24 hours or a goal urine output of at least $0.5 \mathrm{ml} / \mathrm{kg} / \mathrm{hr}$.

- Lactated Ringer's solution should be used as the resuscitation fluid of choice.

Now commonly recognized as the primary form of initial management, the importance of adequate fluid resuscitation cannot be understated. In severe cases, acute pancreatitis can lead to pancreatic necrosis and ongoing pancreatic enzyme release [12]. One of the triggers of necrosis is thought to be inadequate glandular perfusion. The pancreatic microcirculation encompasses the celiac and superior mesenteric arteries that branch off to supply the pancreatic acinus. Acute pancreatitis invokes a state of hypovolemia, causing a combination of microangiopathic effects and pancreatic edema that decreases blood flow. This disruption in perfusion may be an important factor responsible for the transition from mild, interstitial edematous disease to severe, necrotizing pancreatitis [13-17]. Current proposed mechanisms of this pathophysiology include hypercoagulability with microthrombi, endothelial damage from free radicals, increased capillary permeability, and hypovolemia [18]. The resultant ischemia produces a flush of cytokines and inflammatory mediators, which can progress into 
the development of the systemic inflammatory response syndrome (SIRS) and pancreatic necrosis and eventually lead to persistent ( $>48$ hour) organ failure.

As the above data suggests, inadequate fluid resuscitation leading to poor pancreatic microcirculatory perfusion has been associated with acute necrotizing pancreatitis [19]. Specifically, we now know that early fluid resuscitation has more of a therapeutic effect than delayed fluid resuscitation. In one study evaluating specifically the time course of IV hydration, early was defined as receiving greater than one-third of the total 72 hours fluid volume within the first 24 hours of hospitalization; whereas, late was defined as receiving less than one-third of the total volume [20]. While the investigation did not focus on the total infused fluid volume, it concluded that the group receiving early fluid resuscitation experienced less mortality than those receiving later resuscitation. Other studies have since supported this claim, including a retrospective analysis of 436 acute pancreatitis patients, which found an association between early fluid resuscitation and decreased SIRS, organ failure at 72 hours, length of hospital stay, and a lower rate of ICU admission [21].

Though early fluid resuscitation is generally agreed upon as an intervention of paramount importance, there are currently no standard guidelines on the optimal fluid type, volume, rate, or duration of treatment [22]. Though human studies regarding the rate of hydration consistently show decreased morbidity and mortality with aggressive hydration in the first 24 hours, the total volume of hydration at the 48-hour mark seems to have a limited effect on patient outcomes. The current American College of Gastroenterology (ACG) guidelines have recommended $250-500 \mathrm{~mL}$ per hour of isotonic crystalloid solution in the first $12-24$ hours with frequent re-evaluation every 6 hours, with an ultimate goal of decreasing the BUN levels [23]. Some experts recommend that in addition to the 1-2 L fluid bolus given in the emergency department, the starting infusion should be at a rate of $250-300 \mathrm{ml} / \mathrm{h}$ or enough to produce a urine output of at least $0.5 \mathrm{ml} / \mathrm{kg} / \mathrm{hr}$ [24]. The goal within the first 24 hours is a total infusion volume of 2.5-4 L, with adjustments to be made based on the patient's age, weight, physical exam, and comorbid conditions [25].

The type of resuscitation fluid has not been satisfactorily studied. However, in the most widely cited prospective study of fluid resuscitation in acute pancreatitis, $\mathrm{Wu}$ and colleagues found that the use of Lactated Ringer's solution, as opposed to normal saline, resulted in less SIRS and a decreased c-reactive protein at 48 hours [26]. There have been no other prospective fluid studies evaluating different types of resuscitative fluid, and thus it is generally recommended, in the absence of better evidence, that Lactated Ringer's be used as the resuscitative fluid of choice.

It is also very important to recognize the consequences of over-resuscitation - most notably the development of intra-abdominal compartment syndrome. In a study of patients with predicted severe pancreatitis whose hematocrit was aggressively lowered upon admission, those with aggressive lowering of their hematocrit had greater morbidity and mortality [27]. 


\section{Pharmacologic Strategies}

\section{Antibiotics}

- Not recommended for prophylaxis of infected pancreatic necrosis, though indicated if another source of infection is clinically suspected.

Infected pancreatic necrosis continues to be the most common cause of death in patients with acute pancreatitis who survive the early phase, accounting for up to $70 \%$ of all mortality. Though initially present in about $5 \%$ of patients with acute pancreatitis, pancreatic necrosis puts patients at a very high risk of pancreatic bed infection, occurring in 50-70\% of cases $[2,6]$. Antibiotic prophylaxis and therapy have been a long-contested solution to this problem, with the potential for reduction in the morbidity and mortality associated with severe acute pancreatitis.

Use of antibiotics in previous years as prophylaxis for infected necrosis was recommended and common in practice, supported by early research demonstrating broad-spectrum antibiotics to decrease the rate of infected pancreatic necrosis [28]. A meta-analysis in 2001, which included three randomized controlled trials, compared antibiotic prophylaxis to no prophylaxis in the setting of acute necrotizing pancreatitis [29]. They found a reduction of $21.2 \%$ in sepsis and $12.3 \%$ in mortality in patients receiving prophylactic antibiotics; however, there was no difference in the incidence of pancreatic infection.

Studies since then have continued to have conflicting results, with a meta-analysis published in 2008, which included the same three previously mentioned randomized controlled trials, demonstrating no difference in the rates of pancreatic infection or mortality between the group receiving antibiotics versus placebo [30]. An evaluation of the same trials by a Cochrane review confirmed no difference in mortality but found a significant difference with the use of Imipenem alone in terms of preventing infection [31]. Most recently in 2011, an evaluation of 14 randomized controlled trials totaling 841 patients compared those receiving antibiotics versus placebo. No significant differences were reported in mortality, incidence of infected pancreatic necrosis, non-pancreatic infection, and surgical intervention [32]. There may even be an association with antibiotic use and pancreatic fungal infections [33].

There has been some consideration of using probiotics for prevention of infection in acute pancreatitis; however a meta-analysis in 2009 demonstrated no reduction in the risk of pancreatic infection or associated mortality [34]. There may be some benefit in selective gut decontamination, which is the process of using oral antibiotics to eradicate enteric gramnegative rods and thus reduce bacterial translocation from the GI tract into the pancreas, but further studies need to be performed [35].

Ultimately, prophylactic antibiotics are not recommended for use in acute pancreatitis and should not be administered in the first 24 hours of the episode unless there is clinical suspicion for concurrent extrapancreatic infection. Patients may present initially with sepsis, SIRS, and/or multi-organ failure. Treatment with antibiotics is appropriate if evaluation of the patient, via blood cultures and fine needle aspirations of pancreatic necrosis, reveals 
infection. However, if there is no obvious source of infection, antibiotics should be discontinued [23].

\section{Emerging Pharmacologic Therapies}

- No evidence to suggest that any of the current targeted therapies provide benefit.

There has been extensive research evaluating pharmacologic agents such as somatostatin, octreotide, atropine, glucagon, and cimetidine that specifically reduce pancreatic secretions. Most of the research has had disappointing outcomes. For example, cimetidine, assessed via a meta-analysis of 5 randomized controlled trials in 2002 , has also proven to be no more effective than placebo in decreasing complications or pain $[36,37]$.

Since acute pancreatitis features autodigestion from proteases, protease inhibitors would theoretically provide benefit. However, studies on gabexate mesliate and aprotinin have not demonstrated an improvement in patient outcomes [38,39]. Numerous other attempts at targeted pharmacologic therapy, such as lexipafant (platelet activating factor antagonist), antioxidants, corticosteroids, nitroglycerin, IL-10 or TNF-a antibodies, have shown no benefit in the treatment of acute pancreatitis and should not be used at this time [40].

\section{Nonpharmacologic Strategies}

\section{Nutrition}

- Enteral feeding if tolerated is strongly preferred over parenteral feeding

- Mild acute pancreatitis: start enteral feeds within the first week of hospitalization

- Severe acute pancreatitis: start enteral feeds within the first 72 hours of hospitalization

In the past, patients with acute pancreatitis were kept nothing by mouth - with the intent of providing pancreatic and bowel rest - until pain resolution. The practice did not have any demonstrable benefit as bowel rest is associated with intestinal mucosal atrophy and increased infectious complications due to bacterial translocation [41]. Therefore, in order to maintain gut barrier function, enteral feeding is preferred over parenteral feeding in the management of acute pancreatitis $[42,43]$.

In mild acute pancreatitis, early initiation of oral intake with a low-fat soft solid diet is often tolerated and has been demonstrated to be as equally efficacious as tube feedings [44]. Enteral feeding is recommended within one week of hospitalization, typically after cessation of nausea, vomiting, discontinuation of parenteral analgesics, reduction in abdominal pain, and return of bowel sounds [45].

In patients with severe acute pancreatitis, it is recommended to initiate enteral nutrition via nasoenteric tubes within the first 72 hours of hospitalization. A 2012 meta-analysis of 381 patients with severe acute pancreatitis confirms the benefit of enteral vs. parenteral feeds. With two groups randomized to receive each variation of nutrition, those with enteral feeds benefitted in mortality, infection, organ failure, and had a lower surgical rate [46]. Nasojejunal feeding has long been preferred, although there is evidence that nasogastric 
have similar clinical efficacy [47]. Although evidence shows a preference towards enteral feeding, should the patient be unable to tolerate it, or not meet nutritional goals, parenteral nutrition should be initiated while maintaining a slow rate of enteral feeds [23].

\section{Management of Underlying Etiology}

- Evaluate for gallstones with abdominal ultrasound in all acute pancreatitis patients

- Cholecystectomy is recommended for those with acute pancreatitis and gallstones found on imaging.

- ERCP should not be used in acute pancreatitis unless there is ascending cholangitis or clinical decompensation in the setting of elevated liver tests

Identifying and treating the underlying etiology remains the most effective means of preventing a recurrence of acute pancreatitis. The most common cause is gallstones (40-70\%) followed by alcohol use (25-35\%) [48-50]. For this reason, an abdominal ultrasound is recommended for all those presenting with acute pancreatitis to evaluate for gallstones; should they be present, an elective cholecystectomy is suggested prior to discharge [23].

In patients with acute biliary pancreatitis, endoscopic retrograde cholangiopancreatogrpahy (ERCP) should only be used in the context of ascending cholangitis, or worsening liver tests with concomittant clinical deterioration [23,51]. In general therefore, ERCP should be avoided in the management of acute pancreatitis. The role of ERCP in gallstone pancreatitis is discussed in detail elsewhere in this volume.

\section{Recurrence}

The recurrence rate of acute pancreatitis is roughly $25 \%$ but can be up to $50 \%$ in patients whose predisposing factors have not been identified or addressed [52]. As stated above, identifying and treating the underlying etiology is the most important step in preventing recurrence. In the case of a recurrent episode, additional imaging modalities are recommended to evaluate the anatomy of the region and possibly biliary or pancreatic ductal sludge as well as obstructive calcifications. A computed tomorpgraphy scan, magnetic resonance cholangiopancreatography exam or endoscopic ultrasound may be employed to visualize the area effectively [23].

\section{Summary}

While there continues to be high mortality and morbidity associated with acute pancreatitis, treatment remains largely supportive with early aggressive intravenous fluid resuscitation in the first 24 hours used to maintain the pancreatic microcirculation and prevent progression to severe acute pancreatitis, SIRS, multi-organ failure, and pancreatic necrosis. Optimal type, volume, and rate of infusion for the IV fluids require further randomized controlled trials. No pharmacologic therapies have been shown to be of benefit in reducing the risk of these devastating complications. Furthermore, antibiotics are not recommended for prophylaxis of infected pancreatic necrosis and have shown no benefit in multiple large- 
scale studies. Identifying and treating the underlying etiology is the best way to prevent recurrence.

\section{References}

1. Neoptolemos JP, Raraty M, Finch M, et al. Acute pancreatitis: the substantial human and financial costs. Gut. 1998; 42:886-91. [PubMed: 9691932]

2. Mann DV, Hershman MJ, Hittinger R, et al. Multicentre audit of death from acute pancreatitis. Br J Surg. 1994; 81:890-3. [PubMed: 8044613]

3. Russo MW, Wei JT, Thiny MT, et al. Digestive and liver disease statistics, 2004. Gastroenterology. 2004; 126:1448-53. [PubMed: 15131804]

4. Fagenholz PJ, Castillo CF, Harris NS, et al. Increasing United States hospital admissions for acute pancreatitis, 1988-2003. Ann Epidemiol. 2007; 17:491-97. [PubMed: 17448682]

5. Peery AF, Dellon ES, Lund J, et al. Burden of gastrointestinal disease in the United States: 2012 update. Gastroenterology. 2012; 132:1179-87. [PubMed: 22885331]

6. Appelros S, Lindgren S, Borgström A. Short and long term outcome of severe acute pancreatitis. Eur J Surg. 2001; 167:281-6. [PubMed: 11354320]

7. Banks PA, Bollen TL, Dervenis C, et al. Classification of acute pancreatitis 2012: revision of the Atlanta classification and definitions by international consensus. Gut. 2013; 62:102-11. [PubMed: 23100216]

8. Baillargeon JD, Orav J, Ramaqopal V, et al. Hemoconcentration as an early risk factor for necrotizing pancreatitis. Am J Gastroenterol. 1998; 93:2130-4. [PubMed: 9820385]

9. Brown A, Orav J, Banks PA. Hemoconcentration is an early marker for organ failure and necrotizing pancreatitis. Pancreas. 2000; 20:367-72. [PubMed: 10824690]

10. Muddana V, Whitcomb DC, Khalid A, et al. Elevated serum creatinine as a marker of pancreatic necrosis in acute pancreatitis. Am J Gastroenterol. 2009; 104:164-70. [PubMed: 19098865]

11. Wu BU, Bakker OJ, Papachristou GI, et al. Blood urea nitrogen in the early assessment of acute pancreatitis. Arch Intern Med. 2011; 171:669-76. [PubMed: 21482842]

12. Takeda K, Mikami Y, Fukuyama S, et al. Pancreatic ischemia associated with vasospasm in the early phase of human acute necrotizing pancreatitis. Pancreas. 2005; 30:40-9. [PubMed: 15632698]

13. Hotz HG, Foitzik T, Rohweder J, et al. Intestinal microcirculation and gut permeability in acute pancreatitis: early changes and therapeutic implications. J Gastrointest Surg. 1998; 2:518-25. [PubMed: 10458730]

14. Banks PA, Freeman ML. Practice guidelines in acute pancreatitis. Am J Gastroenterol. 2006; 101:2379-2400. [PubMed: 17032204]

15. Knoefel WT, Kollias N, Warshaw AL, et al. Pancreatic microcirculatory changes in experimental pancreatitis of graded severity in the rat. Surgery. 1994; 116:904-13. [PubMed: 7940196]

16. Strate T, Mann O, Kleinhans H, et al. Microcirculatory function and tissue damage is improved after therapeutic injection of bovine hemoglobin in severe acute rodent pancreatitis. Pancreas. 2005; 30:254-9. [PubMed: 15782104]

17. Bassi D, Kollias N, Fernandez-del Castillo C, et al. Impairment of pancreatic microcirculation correlates with the severity of acute experimental pancreatitis. J Am Coll Surg. 1994; 179:257-63. [PubMed: 8069418]

18. Cuthbertson CM, Christophi C. Disturbances of the microcirculation in acute pancreatitis. Brit J Surg. 2006; 93:518-30. [PubMed: 16607683]

19. Brown A, Baillargeon JD, Hughes MD, et al. Can fluid resuscitation prevent pancreatic necrosis in severe acute pancreatitis? Pancreatology. 2002; 2:104-7. [PubMed: 12123089]

20. Gardner TB, Vege SS, Chari ST, et al. Faster rate of initial fluid resuscitation in severe acute pancreatitis diminishes in-hospital mortality. Pancreatology. 2009; 9:770-6. [PubMed: 20110744]

21. Warndorf MG, Kurtzman JT, Bartel MJ, et al. Early fluid resuscitation reduces morbidity among patients with acute pancreatitis. Clin Gastroenterol Hepatol. 2011; 9:705-9. [PubMed: 21554987] 
22. Nasr JY, Papachristou GI. Early fluid resuscitation in acute pancreatitis: a lot more than just fluids. Clin Gastroenterol and Hepatol. 2011; 9:633-4.

23. Tenner S, Baillie J, et al. American College of Gastroenterology Guideline: Management of Acute Pancreatitis. Am J Gastroenterol. 2013; 108:1400-1415. [PubMed: 23896955]

24. Talukdar R, Vege SS. Early management of severe acute pancreatitis. Curr Gastroenterol Rep. 2011; 13:123-30. [PubMed: 21243452]

25. Besselink M, van Santvoort H, Freeman M, et al. IAP/APA evidence-based guidelines for the management of acute pancreatitis. Pancreatology. 2013; 13:e1-e15. [PubMed: 24054878]

26. Wu BU, Hwang JQ, Gardner TB, et al. Lactated Ringer's solution reduces systemic inflammation compared with saline in patients with acute pancreatitis. Clin Gastroenterol Hepatol. 2011; 9(8): 710-717. [PubMed: 21645639]

27. Mao EQ, Fei J, Peng YB, et al. Rapid hemodilution is associated with increased sepsis and mortality among patients with severe acute pancreatitis. Chin Med J. 2010; 123(13):1639-44. [PubMed: 20819621]

28. Heinrich S, Schäfer M, Rousson V, et al. Evidence-based treatment of acute pancreatitis: a look at established paradigms. Ann Surg. 2006; 243:154-68. [PubMed: 16432347]

29. Sharma VK, Howden CW. Prophylactic antibiotic administration reduces sepsis and mortality in acute necrotizing pancreatitis: a meta-analysis. Pancreas. 2001; 22:28-31. [PubMed: 11138967]

30. Bai Y, Gao J, Zou DW, et al. Prophylactic antibiotics cannot reduce infected pancreatic necrosis and mortality in acute necrotizing pancreatitis: evidence from a meta-analysis of randomized controlled trials. Am J Gastroenterol. 2008; 103:104-10. [PubMed: 17925000]

31. Villatoro E, Mulla M, Larvin M. Antibiotic therapy for prophylaxis against infection of pancreatic necrosis in acute pancreatitis. The Cochrane Database of Syst Rev. 2010; (5):CD002941. [PubMed: 20464721]

32. Wittau M, Mayer B, Scheele J, et al. Systematic review and meta-analysis of antibiotic prophylaxis in severe acute pancreatitis. Scand J Gastroenterol. 2011; 46:261-70. [PubMed: 21067283]

33. Trikudanathan G, Navaneethan U, Vege SS. Intra-abdominal fungal infections complicating acute pancreatitis: a review. Am J Gastroenterol. 2011; 106:1188-92. [PubMed: 21731015]

34. Sun S, Yang K, He X, et al. Probiotics in patients with severe acute pancreatitis: a meta-analysis. Langenbecks Arch Surg. 2009; 394:171-7. [PubMed: 18633636]

35. Luiten EJ, Hop WC, Lange JF, et al. Controlled clinical trial of selective decontamination for the treatment of severe acute pancreatitis. Ann Surg. 1995; 222:57-65. [PubMed: 7618970]

36. Morimoto T, Noguchi Y, Sakai T, et al. Acute pancreatitis and the role of histamine- 2 receptor antagonists: a meta-analysis of randomized controlled trials of cimetidine. Euro J of Gastroenterol Hepatol. 2002; 14:679-86.

37. Uhl W, Buchler MW, Malfertheiner P, et al. A randomized, double blind, multicentre trial of octreotide in moderate to severe acute pancreatitis. Gut. 1999; 45:97-104. [PubMed: 10369711]

38. Andriulli A, Leandro G, Clemente R, et al. Meta-analysis of somatostatin, octreotide and gabexate mesilate in the therapy of acute pancreatitis. Aliment Pharmacol Ther. 1998; 12:237-45. [PubMed: 9570258]

39. Heinrich S, Schäfer M, Rousson V, et al. Evidence-based treatment of acute pancreatitis: a look at established paradigms. Ann Surg. 2006; 243:154-68. [PubMed: 16432347]

40. Bang UC, Semb S, Nojgaard C, et al. Pharmacological approach to acute pancreatitis. World J Gastroenterol. 2008; 14:2968-76. [PubMed: 18494044]

41. Steinberg W, Tenner S. Medical progress: acute pancreatitis. New Engl J Med. 1994; 330:1198210. [PubMed: 7811319]

42. Petrov MS, Kukosh MV, Emelyanov NV. A randomized controlled trial of enteral versus parenteral feeding in patients with predicted severe acute pancreatitis shows a significant reduction in mortality and in infected pancreatic complications with total enteral nutrition. Dig Surg. 2006; 23:336-45. [PubMed: 17164546]

43. Gupta R, Patel K, Calder PC, et al. A randomised clinical trial to assess the effect of total enteral and total parenteral nutritional support on metabolic, inflammatory and oxidative markers in patients with predicted severe acute pancreatitis II (APACHE 6). Pancreatology. 2003; 3:406-13. [PubMed: 14526151] 
44. Eckerwall GE, Tingstedt BB, Bergenzaun PE, et al. Immediate oral feeding in patients with acute pancreatitis is safe and may accelerate recovery--a randomized clinical study. Clin Nutr. 2007; 26:758-63. [PubMed: 17719703]

45. Jacobson BC, Vandr Vliet MB, Hughes MD, et al. A prospective, randomized trial of clear liquids versus low-fat solid diet as the initial meal in mild acute pancreatitis. Clin Gastroenterol Hepatol. 2007; 5:946-51. [PubMed: 17613280]

46. Yi F, Ge L, Zhao J, et al. Meta-analysis: total parenteral nutrition versus total enteral nutrition in predicted severe acute pancreatitis. Intern Med. 2012; 51:523-30. [PubMed: 22449657]

47. Eatock FC, Chong P, Menezes N, et al. A randomized study of early nasogastric versus nasojejunal feeding in severe acute pancreatitis. Am J Gastroenterol. 2005; 100:432-9. [PubMed: 15667504]

48. Lankisch PG, Assmus C, Lehnick D, et al. Acute pancreatitis: does gender matter? Dig Dis Sci. 2001; 46:2470-4. [PubMed: 11713955]

49. Gullo I, Migliori M, Olah A, et al. Acute pancreatitis in five European countries: etiology and mortality. Pancreas. 2002; 24:223-7. [PubMed: 11893928]

50. Lowenfels AB, Maisonneuve P, Sullivan T. The changing character of acute pancreatitis: epidemiology, etiology, and prognosis. Curr Gastroenterol Rep. 2009; 11:97-103. [PubMed: 19281696]

51. Freeman ML, DiSario JA, Nelson DB, et al. Risk factors for post-ERCP pancreatitis: a prospective, multicenter study. Gastrointest Endosc. 2001; 54:425-34. [PubMed: 11577302]

52. Andersson R, Andersson B, et al. Incidence, management and recurrence rate of acute pancreatitis. Scand J Gastroenterol. 2004; 39(9):891-4. [PubMed: 15513389] 


\section{Key points}

- Early aggressive fluid resuscitation with lactated Ringer's with a goal total infusion of 2.5-4 L in the first 24 hours.

- Antibiotics are not recommended for prophylaxis of infected pancreatic necrosis, though indicated if another source of infection is clinically suspected.

- Enteral feeding if tolerated is strongly preferred over parenteral feeding, especially in severe acute pancreatitis.

- ERCP in acute gallstone pancreatitis should not be performed unless there is evidence of ascending cholangitis or there is clinical deterioration in the context of rising liver tests.

- Cholecystectomy is recommended prior to discharge for those with acute pancreatitis and gallstones found on imaging. 